\title{
An Approach based on Run Length Count for Denoising the Kannada Characters
}

\author{
Karthik S \\ Department of ISE \\ PES Institute of Technology \\ Bangalore South Campus
}

\author{
Mamatha H.R \\ Department of ISE \\ PES Institute of Technology \\ Bangalore West Campus
}

\author{
Srikanta Murthy K \\ Department of CSE \\ PES Institute of Technology \\ Bangalore South Campus
}

\begin{abstract}
Optical Character Recognition (OCR) is one of the important fields in image processing and pattern recognition domain. OCR with high accuracy finds application in offices, banks, healthcare etc. The accuracy of the OCR is primarily dependent on the quality of the input image. So, to achieve high accuracy OCR we should provide a high quality image, which is free from different types of noises, degradation, skews etc. In this paper, we have made an attempt to remove the noise, which is present in the input image. A novel method based on run length count is proposed to denoise the images. In this approach first the noisy image is binarized. Based on the horizontal and vertical run length count, the noise in the image will be identified and eliminated. The algorithm is tested with noisy epigraphical document images, noisy printed document images. The effectiveness of the algorithm is verified with images having synthetic noise derived from Gaussian, Speckle and Poisson noise models. The experimental results show that the proposed method is efficient for noise elimination.
\end{abstract}

\section{Keywords}

Optical Character Recognition, Image Denoising, Run Length Count, Epigraphical Document, Printed Document, Gaussian Noise, Speckle Noise, Poisson Noise

\section{INTRODUCTION}

The character recognition is an important area of research for its applications in post office, banks and other organizations. Hence, it has received extensive attention for many years in academic and production fields. Still today the character recognition is an open challenge because of the need to achieve high precession and accuracy. The accuracy of the OCR is highly dependent on the quality of the image [1]. Ideally, OCR expects the image free from all types of noises, skew, smear, smudge etc. But in practice it is not achieved due to malfunction in various external agents such as data transmission media, image quantization process, data acquisition tools etc. Because of these problems there is a clear need to preprocess the image before proceeding to recognition phase. Thinning and smoothing are the two important operations to be considered in preprocessing stage. Smoothing operation is used to eliminate unwanted noise in the image like smear, smudge and other degraded noises. Here we have focused our work only to eliminate the noise, which are present in the image. Image denoising is a challenging job because noise removal might introduce artifacts and blur the image.

A detailed survey about various denoising techniques is reported by Mukesh $\mathrm{C}$ Motwani et al. [2]. They have classified the denoising techniques into spatial filtering and Transform domain filtering. The spatial filtering technique discusses the use of commonly used filters like mean, median etc. The Transform domain filtering is mainly based on Fast Fourier transforms, wavelet transforms and curvelet transforms. Various wavelet based image denoising techniques are presented in [3-5]. Lakhwinder Kaur et al. [3] presented a wavelet based generalized Gaussian distribution model. Mohesen et al. [5] presented a fractal wavelet image denoising technique, which produced highly comparable results. Two new methods of image denoising based on ridgelet and curvelet was proposed in [6,7]. It is claimed that these methods are superior compared to the methods based on wavelets. It is also mentioned that these methods can also be applied to remote sensing images. Sathish et al. [8] proposed denoising technique based on contourlets and experimented with the medical images acquired by Magnetic Resonance Imaging (MRI) and claimed that it achieves higher peak signal to noise ratio (PSNR) when compared to wavelet based methods. A new approach based on cellular neural network to denoise an image was proposed in [9]. This approach is claimed to be effective even under a highly noisy condition. Vijaya and Vasudevan attempted image denosing method based on bilateral filtering and fuzzy clustering [10]. Lei Zhang et al. presented an effective image denoising technique based on principle component analysis with local pixel grouping in [11] which was able to achieve highly competitive results. Antoni Buades et al. presented a method based on non-local mean and achieved satisfactory results $[12,13]$. However, all these approaches focus on eliminating noise in natural images and very less work can be tracked towards the elimination of noise in document image processing. Clearly this warrants a focused work in this direction.

Here, we present a novel method based on the run length count method to eliminate these unwanted noises. In section

2 , we have discussed the background concepts and in section 3 , the proposed technique is discussed. Section 4 presents the outcomes of the experiments and the conclusion is presented in section 5 .

\section{BACKGROUND CONCEPTS}

Run Length encoding is one of the oldest, simple loss less data compression algorithm primarily used to compress the binary graphical data. In this method, any sequence of identical symbols will be counted and replaced by its count. This count is called Run Length Count (RLC). It is noted that this method works well when we have a series of one type of symbol followed by another type.

In binary images the change of one sequence of symbols to another sequence generally indicates the change in the boundary of the object. If we apply this concept to the document images then it indicates edge information of a character. This concept was used to recognize the characters 
in [14]. In this paper, we have used this concept for remove the unwanted noise pixels from the given document images. In this approach, we calculate the horizontal and vertical run length count. To calculate the Horizontal Run Length Count (HRLC), we calculate the continuous sequence of 1's row wise and the same will be done column wise to get the Vertical Run Length Count (VRLC). The next example illustrates the procedure to calculate the vertical run length count.

\begin{tabular}{|l|l|l|l|l|l|l|}
\hline 0 & 1 & 1 & 1 & 0 & 0 & \\
\hline 0 & 0 & 0 & 1 & 1 & 0 & \\
\hline 1 & 1 & 1 & 0 & 0 & 0 & \\
\hline $\mathbf{0}$ & $\mathbf{0}$ & $\mathbf{0}$ & $\mathbf{2}$ & $\mathbf{0}$ & $\mathbf{0}$ & $\longleftarrow$ VRLC \\
\hline
\end{tabular}

\section{PROPOSED METHOD}

The input image is a scanned image contains some noise pixels. Mathematically it can be represented as

$\mathrm{G}(\mathrm{x}, \mathrm{y})=\mathrm{F}(\mathrm{x}, \mathrm{y})+\mathrm{N}(\mathrm{x}, \mathrm{y})$ Equation 1

Where $F(x, y)$ represents the image pixels and $N(x, y)$ indicates the noise function. $\mathrm{G}(\mathrm{x}, \mathrm{y})$ is the combination of image pixels and noise, which we call as distorted image. The aim of this paper is to reduce the noise, which is present in the distorted image. The distorted image will be first binarized using the threshold, which is determined by the Otsu's method [15]. In the binarised image black and white pixels are represented by 0 's and 1's respectively. The black pixel represents the background and white pixel represents the object respectively. Next, we calculate the horizontal and vertical run length count by the approach suggested in the previous section. If they are less than a specified threshold then it is assumed to be noise and will be eliminated. This assumption is very valid as the character data always spread across multiple pixels. The proposed method is summarized in the below algorithm.

The proposed algorithm:

Input: A Image with noise.

Output: A binary image without noise.

Method:

Step 1: Binarise the image using Otsu's threshold

Step 2: For each pixel $\mathrm{p}(\mathrm{x}, \mathrm{y})$ calculate the horizontal and vertical run length count.

Step3: if horizontal and vertical run length count is less than Threshold then

$$
\text { Set } \mathrm{p}(\mathrm{x}, \mathrm{y}) \text { to background pixel }
$$

Else

$$
\text { Retain the } \mathrm{p}(\mathrm{x}, \mathrm{y}) \text { content }
$$

Step 4:. End

For the experimentation, we have used naturally noise effected images and images with synthetic noise. In order to introduce noise in an image, we have used Gaussian noise model, which is derived from equation 2

$\mathrm{G}=\mathrm{F}+\mathrm{g} * \mathrm{~F}$

Equation 2
Where $\mathrm{F}$ is the image data, $\mathrm{g}$ is gaussian random variable given by equation 3

$$
g(n)=\left(1 / \sqrt{\pi \sigma^{2}}\right) * e^{-n^{2} / \sigma^{2}}
$$

Another type of noise model called Poisson noise model, which is derived through the equation 4 , is used to introduce poisson noise.

$\mathrm{G}=\mathrm{F}+\mathrm{p} * \mathrm{~F}$

Equation 4

Where $\mathrm{F}$ is the image data, $\mathrm{p}$ is poisson variable given by equation 5 with mean $\lambda=10$

$$
p(n)=e^{-\lambda} \lambda^{n} / n !
$$

Equation 5

Equation 6 describes the third noise model called speckle noise model. Our approach was tested with samples affected by this type of noises. Speckle noises are commonly observed in image obtained via MRI machines.

$\mathrm{G}=\mathrm{F}+\mathrm{n} * \mathrm{~F}$

Equation 6

Where $\mathrm{F}$ is the image data, $\mathrm{n}$ is uniformly distributed random noise with mean 0 and variance $\mathrm{v}$.

\section{EXPERIMENTAL RESULTS AND DISCUSSIONS}

The proposed method was tested on various noisy images. We have considered three different categories of images i.e. noisy epigraphical document images, images with synthetic noise and printed images with noise. We have used Gaussian, Poisson and Speckle noise models for introducing synthetic noise. Image sample 1,2 and 3 are based on Gaussian, Poisson and Speckle noise models respectively. A few sample of the images before and after noise removal are shown in Fig 1-8. Table 1 depicts the performance of the algorithm on various image samples. Table 2 compares the proposed algorithm with the majority function method and minimum majority function methods[1]. It is found that the proposed method outperformed the existing methods. The comparisons of these methods are shown graphically in fig 9. The algorithm was implemented in Matlab software on a personal computer with Intel Atom Processor with 2 GB RAM.
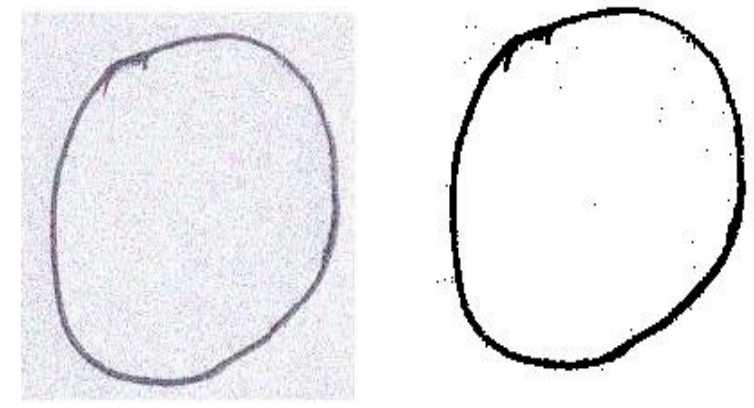

Figure 1: Image sample 1 before and after noise elimination 

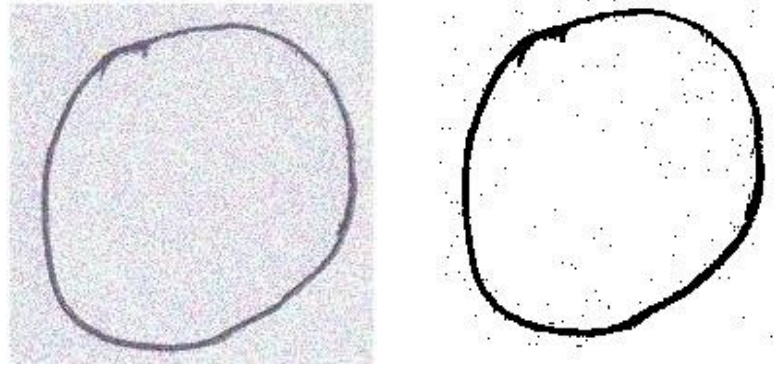

Figure 2: Image sample 2 before and after noise elimination
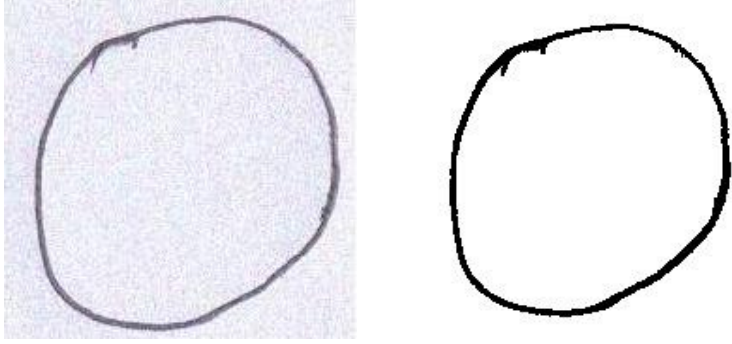

Figure 3: Image sample 3 before and after noise elimination
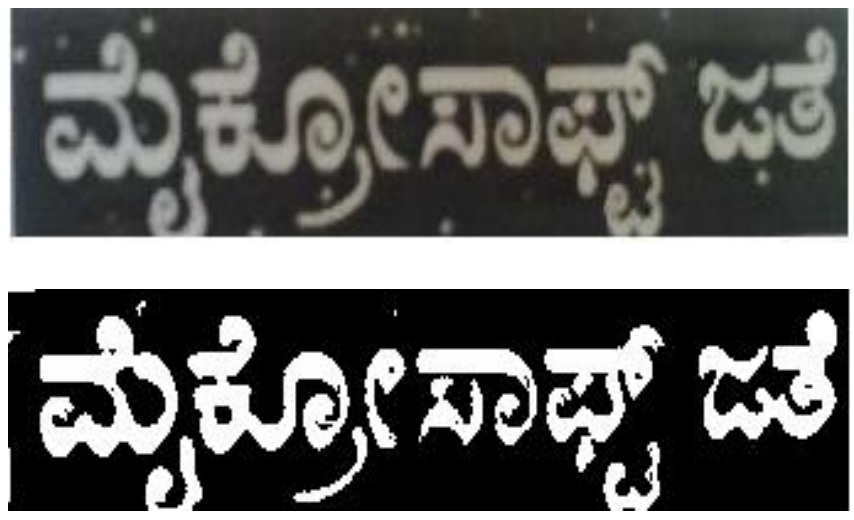

Figure 4: Image sample 4 before and after noise elimination
Table 1: Performance of the proposed method for noise elimination

\begin{tabular}{|c|c|c|c|}
\hline $\begin{array}{l}\text { Image } \\
\text { Name }\end{array}$ & $\begin{array}{c}\text { Total No of } \\
\text { white pixel } \\
\text { before noise } \\
\text { elimination }\end{array}$ & $\begin{array}{c}\text { Total noise } \\
\text { Pixels } \\
\text { Eliminated }\end{array}$ & $\begin{array}{c}\text { Percentage } \\
\text { Of noise } \\
\text { Removed }\end{array}$ \\
\hline Sample 1 & 33959 & 256 & $0.75 \%$ \\
\hline Sample 2 & 33801 & 261 & $0.77 \%$ \\
\hline Sample 3 & 33954 & 236 & $0.70 \%$ \\
\hline Sample 4 & 119138 & 14944 & $12.54 \%$ \\
\hline Sample 5 & 26444 & 4172 & $15.78 \%$ \\
\hline Sample 6 & 13526 & 4031 & $29.80 \%$ \\
\hline Sample 7 & 8394 & 2063 & $24.58 \%$ \\
\hline Sample 8 & 7540 & 2906 & $38.54 \%$ \\
\hline
\end{tabular}

Table 2: Comparative study of existing and proposed method for noise elimination

\begin{tabular}{|l|l|l|l|}
\hline Sample & \multicolumn{1}{|c|}{$\begin{array}{c}\text { Majority } \\
\text { Function } \\
\text { Method }\end{array}$} & $\begin{array}{c}\text { Minimum } \\
\text { Majority } \\
\text { function } \\
\text { method }\end{array}$ & \multicolumn{1}{|c|}{$\begin{array}{c}\text { Proposed } \\
\text { Method }\end{array}$} \\
\hline Sample 5 & $4.64 \%$ & $23.04 \%$ & $15.78 \%$ \\
\hline Sample 6 & $8.31 \%$ & $22.9 \%$ & $29.80 \%$ \\
\hline Sample 7 & $19.99 \%$ & $21.48 \%$ & $24.58 \%$ \\
\hline Sample 8 & $17.26 \%$ & $28.99 \%$ & $38.54 \%$ \\
\hline
\end{tabular}



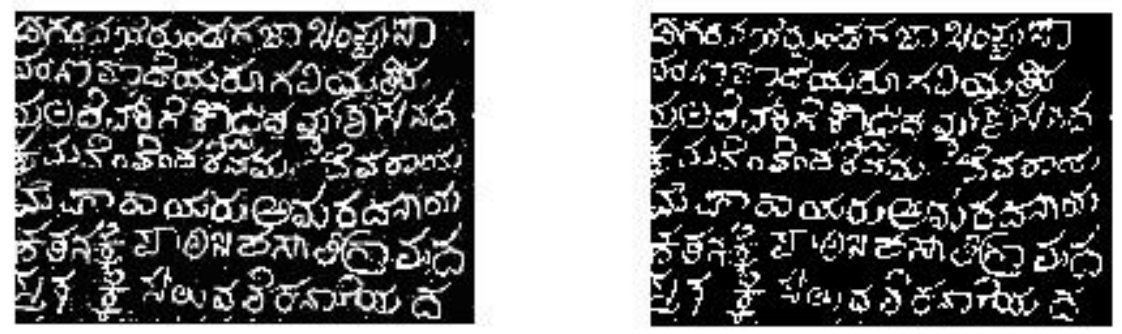

Figure 5: Image sample 5 before and after noise elimination
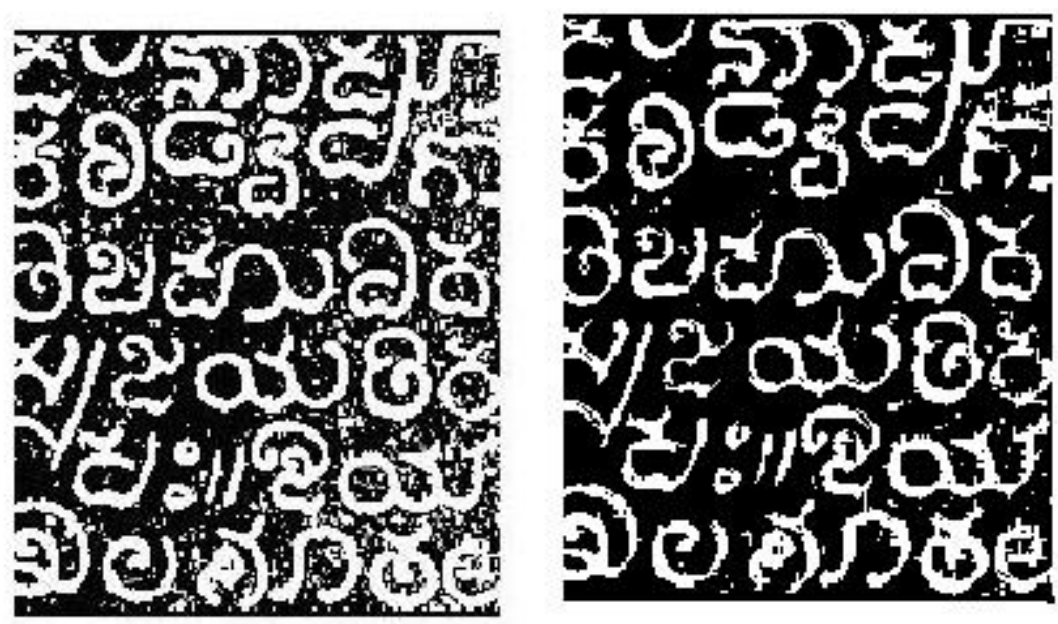

Figure 6: Image sample 6 before and after noise elimination
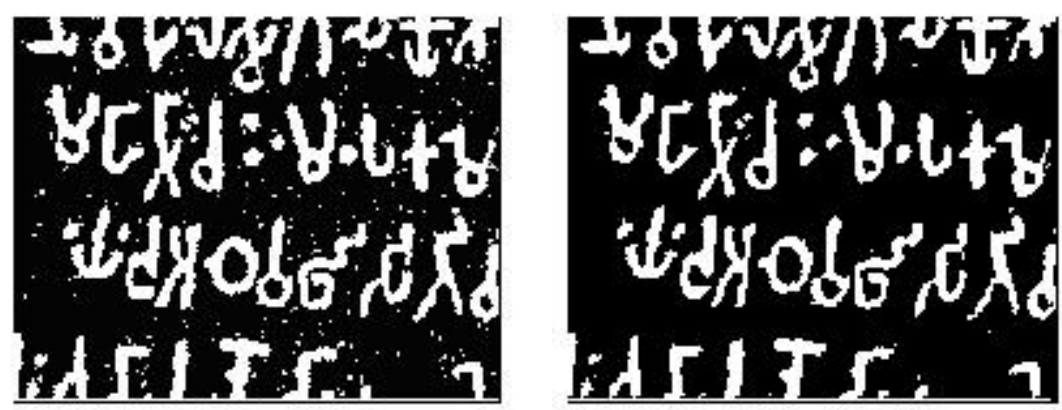

Figure 7: Image sample 7 before and after noise elimination
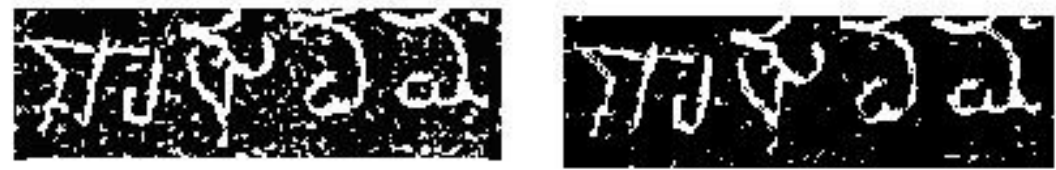

Figure 8: Image sample 8 before and after noise elimination 


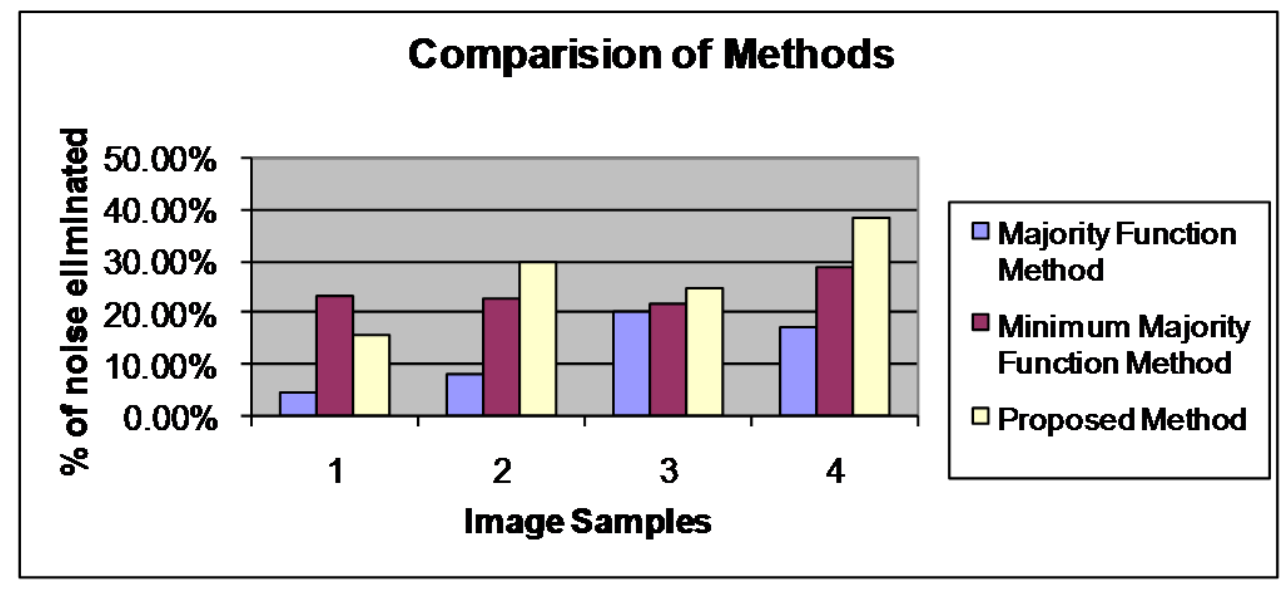

Figure 9: Comparison of the proposed method with the existing methods

\section{CONCLUSION}

The development of high precession optical character recognition system warrants the requirement of a high quality image, which is free from noises and degradations. Hence, noise elimination is one of the vital phases in the OCR development. This paper focuses on development of an effective noise elimination method. Here a novel method based on run length count is proposed to remove the noise from the image. The method has been tested on various image samples, which includes epigraphical document images, printed document images and images with synthetic noise. A comparative study has been made to substantiate the effectiveness of the proposed algorithm. It is observed that the proposed algorithm outperforms the existing methods in removing the noise present in the image.

\section{REFERENCES}

[1] Doreswamy, K.Srikanta Murthy, G.Hemantha Kumar, P.Nagabushan, "Filtering Technique based on Minimum Majority Function to eliminate noises from the Epigraphical Script Images", National Conference on Recent Trends in Information Technology, 2002, page no 35-39

[2] Mukesh Motwani, Mukesh Gadiya, Rakhi Motwani, and Frederick C. Harris, Jr. A Survey of Image Denoising Techniques, Proceedings of GSPx 2004

[3] S. Grace Chang, Bin Yu, Martin Vetterli, "Adaptive Wavelet Thresholding for Image Denoising and Compression", IEEE Transactions On Image Processing, VOL. 9, NO. 9, September 2000, page no: 1532-1546

[4] Lakhwinder Kaur, Savita Gupta, R.C. Chauhan," Image Denoising using Wavelet Thresholding", Indian Conference On Computer Vision, Graphics And Image Processing, 2002

[5] Mohsen Ghazel, George H. Freeman, and Edward R. Vrscay, "Fractal-Wavelet Image Denoising Revisited", IEEE Transactions On Image Processing, VOL. 15, NO. 9, September 2006, page no: 2669-2675

[6] Jean-Luc Starck, Emmanuel J. Candès, and David L. Donoho, "The Curvelet Transform for Image
Denoising", IEEE Transactions On Image Processing, VOL. 11, NO. 6, June 2002, page no: 670-684

[7] JIANG Tao, ZHAO Xin, "Research And Application Of Image Denoising Method Based On Curvelet Transform", The International Archives of the Photogrammetry, Remote Sensing and Spatial Information Sciences. Vol. XXXVII. Part B2, 2008, page no: $363-367$

[8] S.Satheesh, Dr.KVSVR Prasad, "Medical Image Denoising Using Adaptive Threshold Based On Contourlet Transform", Advanced Computing: An International Journal, Vol.2, No.2, March 2011, page no: $52-58$

[9] Tanzila Saba, Amjad Rehman And Ghazali Sulong, “ An Intelligent Approach To Image Denoising", Journal of Theoretical and Applied Information Technology, page no: $32-36$

[10] G. Vijaya, V. Vasudevan," Image Denoising Based On Soft Computing Techniques ", International Journal of Research and Reviews in Applied Sciences, April 2011, page no: $32-37$

[11] Lei Zhang, WeishengDong, DavidZhang, GuangmingShi, "Two-stage image denoising by principal component analysis with local pixel grouping", Pattern Recognition 43, 2010, page no: 1531-1549

[12] Antoni Buades, Bartomeu Coll, Jean-Michel Morel, "A non-local algorithm for image denoising", IEEE Computer Society Conference on Computer Vision and Pattern Recognition, Volume 2, 2005, page no: 60-65

[13] Buades A, B. Coll, J. M. Morel, "A Review Of Image Denoising Algorithms, With A New One", Society for Industrial and Applied Mathematics Multiscale Model. Simulation, Vol. 4, No. 2,2005, page no: 490-530

[14] Mamatha H R, Karthik S, Srikanta Murthy K, "Feature based recognition of handwritten Kannada numerals A comparative study", IEEE International Conference on Computing, Communication and Applications, 2012

[15] Otsu, N., "A Threshold Selection Method from GrayLevel Histograms," IEEE Transactions on Systems, Man, and Cybernetics, Vol. 9, No. 1, 1979, pp. 62-66. 